\title{
CMS Timing Detectors in Phase-2
}

\author{
Francesco Pandolfi* on behalf of the CMS Collaboration \\ INFN Rome \\ E-mail: francesco.pandolfieromal.infn.it
}

\begin{abstract}
To meet the harsh radiation and pile-up environment of HL-LHC, CMS will add a timing layer between the tracker and the calorimeters. The detector will be called MIP Timing Detector (MTD) and will have a target resolution of 30-40 ps at the beginning of data-taking, degrading to only 40-60 ps at the end of operations. The MTD will be comprised of two detectors: a Barrel Timing Layer (BTL) made of scintillating LYSO crystal bars coupled with SiPMs, and an Endcap Timing Layer (ETL) made of Low-Gain Avalanche Diodes (LGADs). Successful R\&D campaigns have been carried out, proving that the required performance is achievable with the chosen technologies.
\end{abstract}

7th Annual Conference on Large Hadron Collider Physics - LHCP2019

20-25 May, 2019

Puebla, Mexico

${ }^{*}$ Speaker. 
The High-Luminosity LHC (HL-LHC) will deliver collisions with instantaneous luminosity of $5.0 \cdot 10^{34} \mathrm{~cm}^{-2} \mathrm{~s}^{-1}$ in the initial phase, and up to $7.5 \cdot 10^{34} \mathrm{~cm}^{-2} \mathrm{~s}^{-1}$ later on. This corresponds to an average number of simultaneous pp collisions (pile up) of 140 and 200, respectively. The challenge for CMS will be to keep the same level of performance as today, in terms of particle reconstruction and identification, in such a high pile up environment.

The CMS global event description relies on efficient track-vertex assignment. It has been found that in events with a vertex linear density (computed along the beam axis) above $1 \mathrm{~mm}^{-1}$ the event reconstruction is degraded. Figure 1 shows the expected linear vertex density for HL-LHC (blue and red curves), and compares it to the current density (green): as can be seen the central part of the luminous region will exceed such threshold.

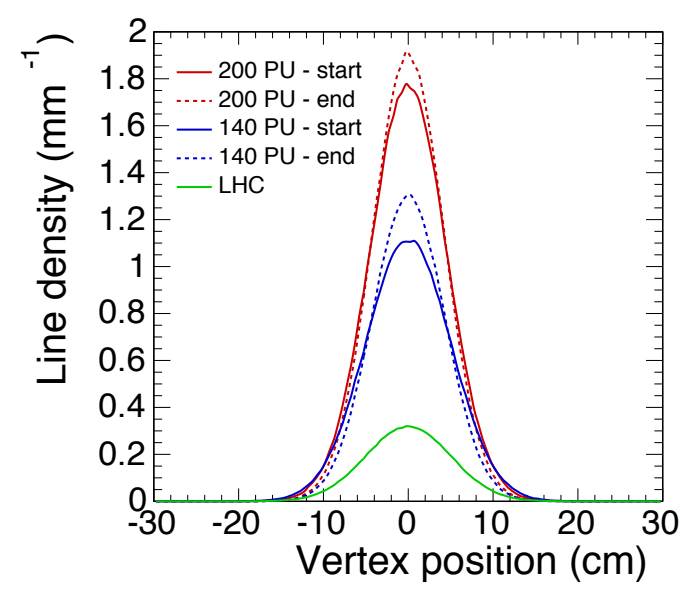

Figure 1: Expected vertex line density for HL-LHC with 200 (red) or 140 (blue) pile up, at the beginning (solid line) or end (dashed line) of runs. The current LHC line density is shown in green as comparison. [1]

In order to mitigate the negative effect of HL-LHC pile up, CMS will add a novel timing detector, placed between the silicon tracker and the calorimeters, with a target resolution of $30-$ $40 \mathrm{ps}$. This would allow to measure, in addition to the spatial position of each vertex, also its timestamp. The luminous region has a spread of about $180 \mathrm{ps}$ : therefore, with good enough timing resolution it would be possible to ignore charged particles which are not compatible with the time of the primary vertex. Table 1 shows how different timing resolutions $\sigma_{t}$ would translate into effective pile up for an ideal detector with $100 \%$ efficiency: a resolution of at least $60 \mathrm{ps}$ is needed in order to have a significant gain.

The MTD will be made of two detectors, with different designs and technologies: the Barrel

Table 1: Effective pile up (PU) for different timing resolutions $\sigma_{t}$ of the MTD. Here an ideal detector with $100 \%$ efficiency is assumed.

\begin{tabular}{lc}
\hline$\sigma_{t}[\mathrm{ps}]$ & Effective PU \\
\hline None & 200 \\
30 & 33 \\
45 & 50 \\
60 & 70 \\
\hline
\end{tabular}



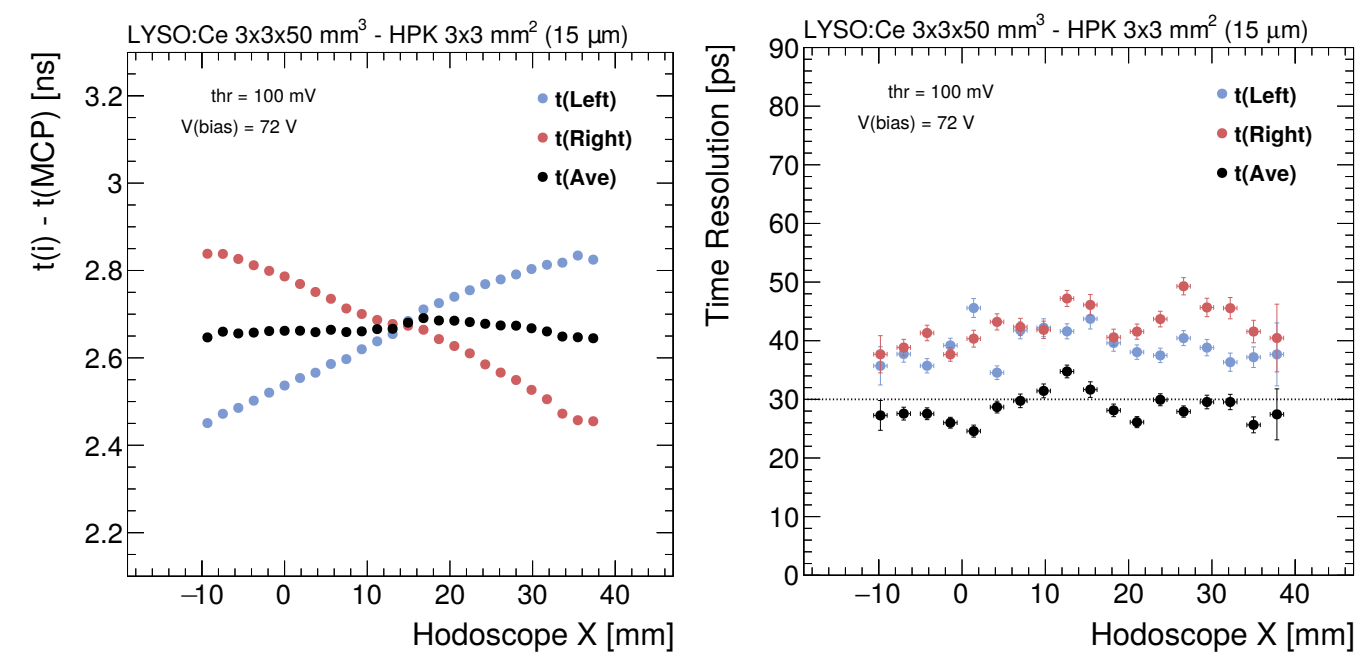

Figure 2: Average timestamp (left) and timing resolution (right) as a function of impact position. [1]

Timing Layer (BTL) will cover the $|\eta|<1.5$ region; the Endcap Timing Layer (ETL) will cover the $1.6<|\eta|<2.9$ region. The BTL will be made of about 300k scintillating LYSO crystal bars, with a front face of $50 \times 3 \mathrm{~mm}^{2}$, aligned in the beam axis direction, and read out by two Silicon PhotoMultipliers (SiPMs) each (one on each end of the bar).

Extensive R\&D campaigns have been carried out in the past years. Some results from a 2018 beam test carried out at CERN, where $80 \mathrm{GeV}$ charged pions were used, are shown in Figure 2. The left plot shows the trend of the timing measurement as a function of the pion impact point along the bar length: the blue and red markers show, respectively, the timestamps recorded in the left and right SiPMs. As expected, if the pion hits closer to the right SiPM, the corresponding timestamp will be smaller (and viceversa) simply because it will take less time for the scintillating photons to reach the silicon detector. Conversely, if one takes the average of the two SiPMs (shown in black), one can see that the measurement does not significantly depend on the impact position. The right plot of Figure 2 shows instead the timing resolution, with the same marker color convention. As can be seen, when averaging the two SiPMS one obtains timing resolutions which are better than the target resolution of $30 \mathrm{ps}$.

It is important to understand the effect of radiation damage on SiPM performance in order to succesfully operate them at HL-LHC. Radiation creates defects in the silicon lattice, which causes dark currents. Part of these defects can be healed by annealing the detector at room temperature, which will be done once a year, during the winter shutdown. The mitigating effect that annealing has on the dark current rate (DCR) is shown in Figure 3 (left), where the DCR is shown as a function of time (the rightmost value on the $x$ axis corresponds to the end of HL-LHC operations, after integrating $4000 \mathrm{fb}^{-1}$ ). In the plot the $\mathrm{BTL}$ is assumed to operate at $-30^{\circ} \mathrm{C}$, and the annealing to take place at $+18^{\circ} \mathrm{C}$. The black line corresponds to no annealing, the blue and green respectively to annealing for two weeks per year and for the full winter shutdowns. As can be seen the DCR is significantly reduced already with only two weeks of annealing per year.

Nevertheless, the DCR will increase throughout HL-LHC operations, meaning that the BTL will increase its power consumption. In order to remain within the power budget, the SiPMs will 

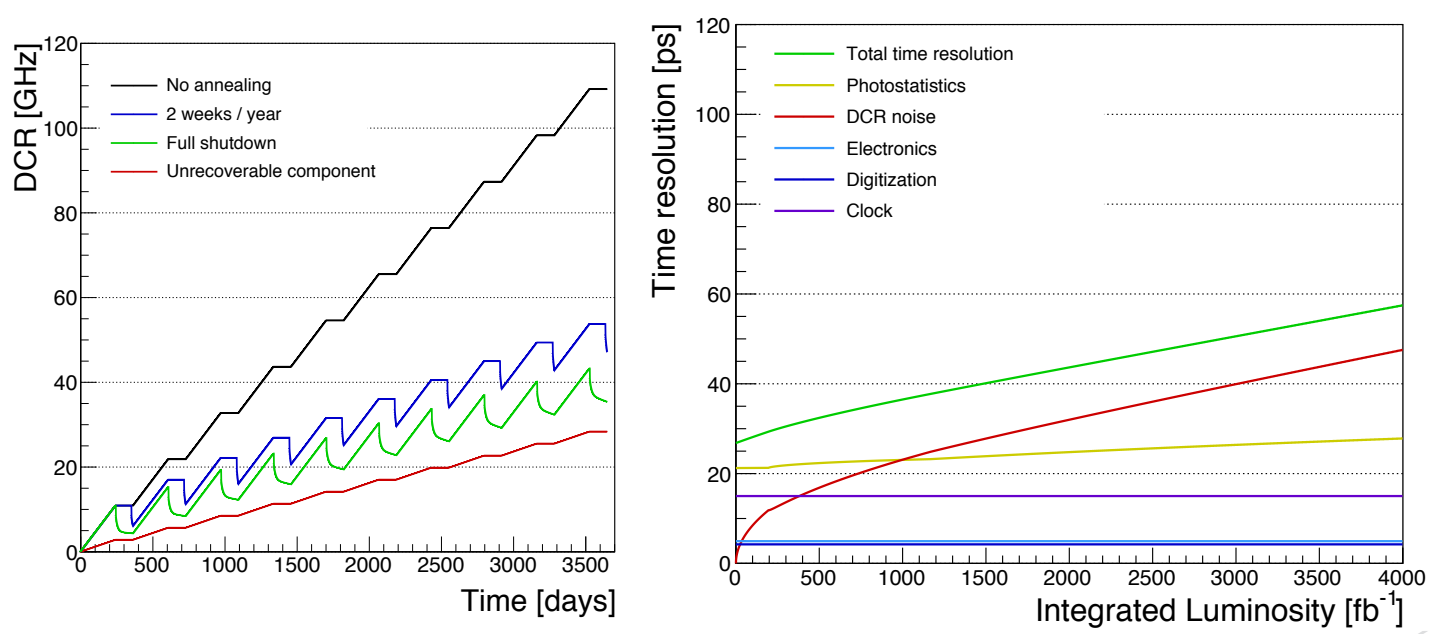

Figure 3: Left: dark current rate (DCR) as a function of time of operation at HL-LHC, for no annealing (black), annealing for two weeks per year (blue) and annealing during the full winter shutdowns (green); here the $\mathrm{BTL}$ is assumed to operate at $-30^{\circ} \mathrm{C}$, and the annealing to take place at $+18^{\circ} \mathrm{C}$. Right: evolution of the BTL timing resolution as a function of time of operation at HL-LHC; the different components are showed in different colors, and the total resolution is shown in green. [1]

have to be operated at a lower gain. And as the photon detection efficiency, and hence the timing resolution performance, depend on the operating bias, the timing resolution is expected to degrade from about 30-40 ps at startup, to about 50-60 ps at the end of HL-LHC operations, with an overall average of 45 ps during HL-LHC data-taking. The trend of the different components which contribute to the BTL timing resolution are shown in Figure 3 (right): the contribution of DCR (red) increases and becomes dominant with increasing time.

Table 2: Expected HL-LHC hadron fluence and radiation dose at different values of $|\eta|$, as expected from a preliminary implementation of the CMS Phase-2 geometry. [2]

\begin{tabular}{ccc}
\hline$|\eta|$ & Hadron Fluence $\left[\mathrm{n}_{\mathrm{eq}} / \mathrm{cm}^{2}\right]$ & Dose $[\mathrm{kGy}]$ \\
\hline 0 & $1.7 \cdot 10^{14}$ & 16 \\
1.15 & $1.9 \cdot 10^{14}$ & 21 \\
1.45 & $2.0 \cdot 10^{14}$ & 25 \\
1.6 & $1.1 \cdot 10^{14}$ & 25 \\
2 & $2.4 \cdot 10^{14}$ & 75 \\
2.5 & $6.6 \cdot 10^{14}$ & 260 \\
3 & $1.7 \cdot 10^{15}$ & 690 \\
\hline
\end{tabular}

Different strategic choices are necessary for the ETL, as it will have to operate in much higher radiation, as can be seen in Table 2, where the expected hadron fluence and radiation dose at different values of $|\eta|$ during HL-LHC operations in shown. As can be seen, while the inner part of the ETL (at $|\eta|=1.6$ ) will sustain similar levels of radiation as the BTL, at higher values of pseudorapidity the dose increases rapidly, up to a factor 30 at $|\eta|=3$. It would be impossible to operate SiPMs in a such high-radiation environment, therefore Low-Gain Avalanche Detectors (LGADs) were chosen, which are silicon devices with a typical internal gain of 10-30. 

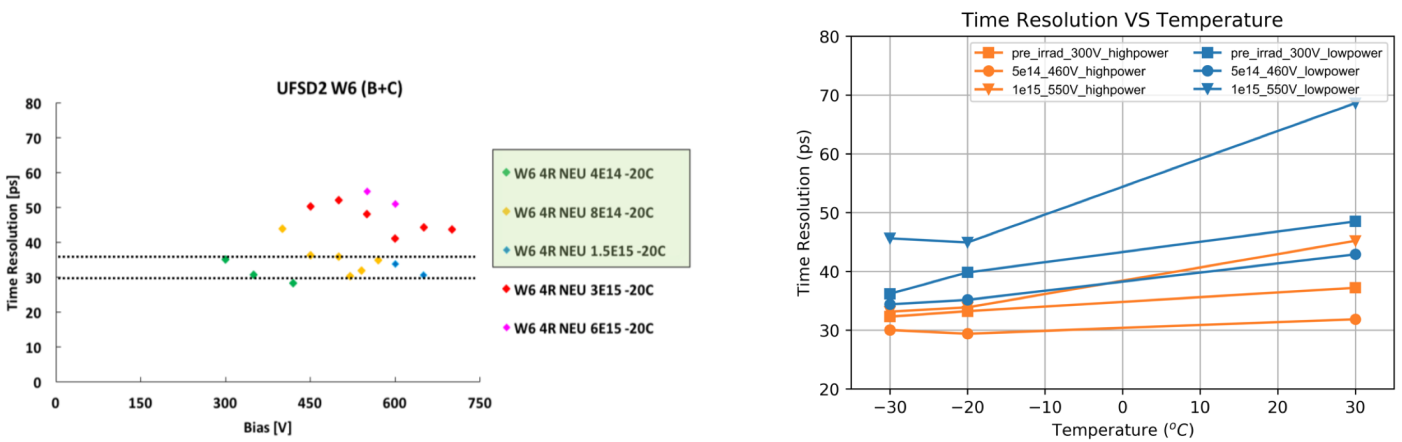

Figure 4: Left: results of irradiation studies on LGADs, shown in terms of timing resolution as a function of operating bias. Different colors correspond to different absorbed doses. Right: simulated time resolution of the LGAD+ASIC as a function of operating temperature. Different markers correspond to different absorbed doses, while the two colors correspond to the two operating modes (high power and low power) of the preamp. [1]

The ETL geometry will consist in two disks of LGADs on each side of the detector, so as to measure each track with two independent hits. This allows to loosen the requirements on the LGADs, in terms of single-hit resolution, to $50 \mathrm{ps}$ (so that the target resolution of 30-40 ps is achieved when combining the two hits).

Irradiation studies have been performed on LGADs, and the results are shown in Figure 4 (left), where the time resolution as a function of the operating bias is shown. Different colors correspond to different absorbed radiation doses: $4 \cdot 10^{14} \mathrm{n}_{\mathrm{eq}} / \mathrm{cm}^{2}$ (shown in green), $8 \cdot 10^{14} \mathrm{n}_{\mathrm{eq}} / \mathrm{cm}^{2}$ (yellow), $1.5 \cdot 10^{15} \mathrm{n}_{\mathrm{eq}} / \mathrm{cm}^{2}$ (blue), and $3 \cdot 10^{15} \mathrm{n}_{\mathrm{eq}} / \mathrm{cm}^{2}$ (red). As can be seen, even in the latter case, which corresponds to a dose equal to about double the total dose of HL-LHC, a timing resolution of about $40-50$ ps can be achieved.

Clearly, it is important to study not only the LGAD resolution, but the expected resolution of the LGAD paired to its ASIC. This has been done with a simulation, and the results are summarized in Figure 4 (right). In the figure the LGAD+ASIC single-hit timing resolution is plotted as a function of the operating temperature. Different marker shapes correspond to different absorbed doses: no irradiation (squares), $5 \cdot 10^{14} \mathrm{n}_{\mathrm{eq}} / \mathrm{cm}^{2}$ (circles), and $1 \cdot 10^{15} \mathrm{n}_{\mathrm{eq}} / \mathrm{cm}^{2}$ (triangles). The two colors, instead, correspond to the two operating modes of the ASIC preamp: low power is shown in blue, and high power in orange. Even after absorbing the full dose of HL-LHC, it seems possible to keep the single-hit resolution to below the threshold of $50 \mathrm{ps}$.

In summary, to meet the harsh radiation and pile-up environment of HL-LHC, CMS will add a timing layer between the tracker and the calorimeters. The detector will be called MIP Timing Detector (MTD) and will have a target resolution of 30-40 ps at the beginning of data-taking, degrading to only 40-60 ps at the end of operations. The MTD will be comprised of two detectors: a Barrel Timing Layer (BTL) made of scintillating LYSO crystal bars coupled with SiPMs, and an Endcap Timing Layer (ETL) made of Low-Gain Avalanche Diodes (LGADs). Successful R\&D campaigns have been carried out, proving that the required performance is achievable with the chosen technologies. 


\section{References}

[1] CMS Collaboration, A MIP Timing Detector for the CMS Phase-2 Upgrade, CMS-TDR-020, submitted.

[2] CMS Collaboration, TECHNICAL PROPOSAL FOR A MIP TIMING DETECTOR IN THE CMS EXPERIMENT PHASE 2 UPGRADE, CERN-LHCC-2017-027, https://cds.cern.ch/record/2296612. 